\title{
Relationship Between Cardiovascular Risk Factors and Periodontal Disease: Current Knowledge
}

\author{
Sergio Granados-Principal ${ }^{1}$, Nuri El-Azem¹, Jose L. Quiles², \\ Patricia Perez-Lopez ${ }^{2}$, Adrian Gonzalez ${ }^{2}$ and MCarmen Ramirez-Tortosa ${ }^{1}$ \\ ${ }^{1}$ Department of Biochemistry and Molecular Biology 2, Institute of Nutrition and Food \\ Technology "José Mataix", Biomedical Research Center, University of Granada, Granada \\ "Department of Physiology, Institute of Nutrition and Food Technology "José Mataix", \\ Biomedical Research Center, University of Granada, Granada,
}

Spain

\section{Introduction}

Periodontitis is a generally chronic disorder characterized by the breakdown of the toothsupporting tissues and the impaired host inflammatory immune response. This condition is due fundamentally to an ecological imbalance between the normal microbial biofilm on teeth and the host tissues. There is increasing evidence linking periodontitis to systemic diseases, such as diabetes, rheumatoid arthritis, and, especially, CVD, hence the search for factors that may explain such relationships. A potential factor which could increase insulin resistance is the production of oxidative stress enhancing ROS in affected periodontal tissues (Bullon et al, 2009).

Metabolic syndrome as originally described is a combination of obesity, hypertension, impaired glucose tolerance or diabetes, hyperinsulinemia, and dyslipidemia (elevated triglycerides and decreased high-density lipoprotein-cholesterol [HDL-C] levels). These same features are also considered as risk factors for atherosclerosis, therefore leading to the deduction that metabolic syndrome constitutes a risk for coronary heart disease. In spite of extensive clinical research on metabolic syndrome, relatively little attention has been directed to its possible relationship to periodontitis. The available data come from epidemiological studies. In a group of 1315 affected individuals (30-92 yrs old), the prevalence of metabolic syndrome was higher among individuals with advanced periodontitis $(66.7 \%)$ than in periodontally healthy individuals (48.8\%) (Borges et al., 2007). Analysis of data from 13,710 participants in the NHANES III (Third National Health and Nutrition Examination Survey) showed a direct relationship between periodontitis and the prevalence of metabolic syndrome (37\% in those with severe periodontitis vs. $18 \%$ in those with mild or no periodontitis), and, particularly, higher prevalence of obesity (48-54\% vs. $31 \%)$, hypertension ( $51-56 \%$ vs. $27 \%$ ), and high glucose levels (18-24\% vs. $8 \%$ ) were stated to be in the moderate to severe periodontitis group compared with the mild periodontitis or periodontally healthy group ( $\mathrm{D}^{\prime}$ Aiuto et al., 2008). 
On the other hand, impaired glucose regulation disorders different from diabetes have been considered in periodontal research, and some encouraging findings have been obtained. Therefore, having deep pockets is significantly associated with impaired glucose tolerance in Japanese non-diabetic subjects and other study in an Israeli non-diabetic adult population revealed a higher occurrence of alveolar bone loss in subjects with elevated fasting glucose level of $\geq 100 \mathrm{mg} / \mathrm{dL}$ (Zadik et al, 2010).

A group of 5,632 participants in the Atherosclerosis Risk in Communities Study were examined in order to establish any relationship between periodontal status, interleukin 1beta (IL-1 $\beta$ ) levels in gingival crevicular fluid (GFC) and HOMA-IR index (Sutherland et al, 2002). An association between periodontal status and the $90^{\text {th }}$ percentile of HOMA-IR was demonstrated, whenever coexisting high GCF IL-1 $\beta$ levels were present. This is an important finding, as IL-1 $\beta$ is related to the pathogenesis of insulin resistance, as well as tumor necrosis factor alpha (TNF-a) (Tilg and Moschen, 2008). Our study supports that a putative relationship between insulin resistance and periodontitis exists. When studied separately, diet can be related to both insulin resistance and periodontitis, nonetheless, we found out that dietary intake seems not to be a determining factor for insulin resistance when it is associated with periodontal disease (Granados-Principal et al, 2011).

Finally, in periodontitis, some perturbation in lipid biomarkers, for example increased total cholesterol in serum and lowdensity lipoprotein cholesterol, has been established. Thus, severe periodontitis is associated with a modest decrease in HDL and LDL cholesterol, and a more robust increase in plasma triacylglycerols. Intensive periodontal therapy results in reductions of total and LDL systemic cholesterols. Nevertheless, the relationship between fatty acids and periodontitis has been demonstrated in only a few studies. Some of these show that n-3 PUFA dietary supplementation modulates alveolar bone resorption following Porphyromonas gingivalis infection in rats and reduces the gingival tissue levels of prostaglandin E2, platelet-activation factor, and leukotriene B4, this being a useful adjunct in the treatment of CP. On the contrary, periodontitis patients with bone loss showed a higher n-6 PUFA plasma level than 27 control subjects. To clarify the situation, we investigated the potential linkage between periodontitis and plasma fatty acids profile, an established cardiovascular disease (CVD) risk factor. Our group has recently demonstrated in 35 years old patients that there is an inter-relationship between periodontitis, plasma fatty acids profile and the increase in metabolic risk factors for cardiovascular diseases (RamirezTortosa et al, 2010). In that paper, the authors found that total plasma fatty acids, saturated, n-6 polyunsaturated and monounsaturated fatty acids, peroxidability index, soluble VCAM, TNF- $\mathrm{a}$, cholesterol, triacylglycerols, and VLDL-c were significantly higher in the periodontitis group compared to the non-periodontitis group. The close association found between plasma triacylglycerols, LDL-c, saturated fatty acids, polyunsaturated fatty acids, total amount of fatty acids and coenzyme Q10 with some periodontal data such as periodontal probing depth, recession of the gingival margin and clinical attachment level (Pearson correlation between 0.3 and 0.6), leads to the conclusion that there is an interrelationship between periodontitis, plasma fatty acids profile and the increase in metabolic risk factors for cardiovascular diseases.

Taking in account all above mentioned the aim of this chapter is to describe the cardiovascular risk factors related with periodontal disease and their relationship because perhaps an interaction between both diseases may result in a worse evolution of them. 


\section{Periodontitis}

\subsection{Introduction}

Tooth structure consists of two different parts: crown, covered with enamel and root, under the gingival line. Dentin comprises most of the tooth parenchyma, and surrounds the pulp chamber, where there are lots of blood vessels and nerves.

Tooth is anchored firmly in the alveolus by the periodontium, a structure that is formed by gums, alveolar bone, tooth cementum and periodontal ligament. The periodontal ligament fixes the tooth concrete to the alveolar bone. Above the ligament, there is a gum fringe, just under the crown. Over the crown base; there is a few millimeters (1 to $3 \mathrm{~mm}$ ) gap of gum that forms a superficial groove, in the border between the gum and the tooth.

Periodontitis is an inflammation and infection of bones and ligaments that act as holders of teeth. It appears when inflammation and infection of gums (gingivitis) is left without treatment or when this treatment is delayed so much time. This inflammation and infection disseminate from gums to ligaments and bones that holds teeth. Due to this loss of support, teeth finally fall out. This problem is infrequent in children, but it's the first cause of dental loss in adults and it affects between $10 \%-15 \%$ of the world population (Baelum \& Lopez, 2004).

Dental plaque and tartar accumulates on the basis of teeth. It can be prevented by adequate tooth cleaning methods and periodic cares from a professional. This inflammation makes that between the gum and teeth, cumulus of tartar and dental plaque are deposited. Continuous inflammation finally develops destruction of tissue and bones surrounding the teeth. Because dental plaque contains germs, it is probably to develop dental abscess, which also contributes to bone destruction. Microorganism at dental plaque releases inflammatory substances that provoke an inflammatory response by immune cells from the host. Leukocytes, mainly neutrophils are recruited by chemotactic stimuli, and they phagocyte and digest this bacteria, preventing them from releasing more inflammatory cytokines. Although this will prevent gingivitis to appear, when neutrophils are overloaded by an excess of bacteria, they degranulate, releasing lots of enzymes and cytokines, aggravating the inflammation and the gingivitis (Kinane, 2001).

It is to say that not all gingivitis progresses to periodontitis and that it does not have to affect every teeth at the same time. Not all periodontitis progress equally in each person, some are more resistant to the development of periodontitis and some are more given to it. Only a few people suffer from advanced tissue destruction around the teeth due to periodontitis and this disease passes with brief episodes of exacerbation and occasional remission. Most people who develop clinical signs of gingivitis do it after 10 - 20 days of plaque accumulation. It appears as redness, swelling and an increased tendency of the gingiva to bleed on gentle probing and it is still reversible to a normal status if the plaque is effectively removed. Periodontitis appears around 6 months before the establishment of gingivitis, but it depends on every single patient (Brecx et al, 1988).

There are several factors that modify the possibility of suffering periodontitis, besides a lack of dental care, such as smoking, drugs (calcium blockers as nifedipine, phenytoin or cyclosporines) hormonal status, stress, age, socioeconomic status and race, systemic diseases, genetics or individual immune response. 


\subsection{Immune response by host cells and inflammation}

Inflammation at the periodontium begins when both bacteria and leukocytes start their fight releasing lots of pro inflammatory factors. The immune response is generated by cell wall components from the bacteria, including lipopolysaccharide (LPS) (Monteiro et al, 2009). Bacteria from the periodontium and some of its components are able to even reach other parts of the organism and produce an inflammatory response there (Tonetti, 2009).

It is now known some of those molecules implicated in the connective tissue destruction, although there are many pathways that correlates between them and participate in this process and we don't know exactly which paper plays every molecule, but the most important involved players are: connective tissue metalloproteinases, reactive oxygen species (ROS) and phagocytosis of matrix components.

Matrix metalloproteinase activity is controlled in vivo through four separate mechanisms: First, they need to be activated by plasmin, trypsin or other proteinases. Second, interleukin1 (IL-1) and transforming growth factor- $\beta$ (TGF- $\beta$ ), induced in inflamed tissues, regulates metalloproteinase production. Third, an a2-macroglobulin serum inhibitor is able to inactivate matrix metalloproteinases and fourth, there is a group of protein inhibitors of matrix metalloproteinases (TIMP) that prevent the conversion of precursor forms of matrix metalloproteinases to their active forms.

Immune response to periodontitis is the key that opens the door to an oxidative stress status and ROS production in the host. Neutrophils are the first line of defense against this infection and one way to achieve their goal is through ROS and reactive nitrogen species (RNS) production by nicotinamide adenine dinucleotide phosphate-oxidase (NADPH oxidase) and nitric oxide synthase (NOS) respectively. Neutrophil NADPH oxidase is a proteic complex normally dissociated until stimulation by protein kinase $C$ (PKC), which phosphorylate p47-phox, a protein that facilitates assembly of NADPH oxidase subunits. PKC is also activated by sn-1, 2-diacylglycerol (DAG). ROS produced then can also act as second messengers activating other pathways, included inflammation, immune response, cell proliferation or apoptosis (Fialkow et al, 2007).

T-cells and B-cells are involved in the defense against periodontitis. Their release products have been detected at the inflammation sites, such as interleukin-2 (IL-2), interleukin-12 (IL12), tumor necrosis factor- $\alpha$ (TNF- $\alpha$ ) and interferon- $\gamma$ from lymphocyte $\mathrm{T}$ helper 1 (Th1); interleukin-4 (IL-4), interleukin-5 (IL-5), interleukin-6 (IL-6), interleukin-10 (IL-10) and interleukin-13 (IL-13) from lymphocyte T helper 2 (Th2) and TGF- $\beta$ from lymphocyte T helper 3 (Th3). T-cells prevail in early stages of the disease while B-cells do it at later stages of periodontitis. It is thought to be a switch between them as the disease gets worse. There is also a great production of immunoglobulin G (IgG) and Immunoglobulin A (IgA) from Bcells (Kiane et al, 2007).

Other cells as macrophages, mononuclear antigen presenting cells, dendritic cells, endothelial cells or adipocytes participate in immune response. These cells possess surface receptors that recognize harmful molecules. Macrophages present a toll-like receptor that recognize lipopolysaccharide (toll-like receptor-4) produced by bacteria in periodontitis, and advanced glycation end products receptor (RAGE) for recognition of advanced glycation end products (AGEs) produced in diabetes. These receptors are able to activate nuclear factor kappa-beta (NF-k $\beta$ ) pathway that promote the expression of inflammatory cytokines (Nassar et al, 2007). 
NF- $\kappa \beta$ pathway modulates a wide variety of gene expressions, but we are interested mostly in its capacity of modulating interleukin-8 (IL-8) expression, which regulates neutrophil migration. Activation of NF- $\kappa \beta$ pathway will be an important contributor to inflammation whereas it is activated. TNF- $\alpha$ is one of the main activators of this pathway. If TNF- $\alpha$ is released, it is surely to have an enhanced NF- $\kappa \beta$ pathway activation. Also IL- $1 \beta$ is able to activate NF- $\kappa \beta$ pathway but in a lesser degree than TNF- $\alpha$ (Fitzgerald et al, 2007).

\subsection{Periodontitis and ROS}

ROS are chemically reactive molecules derived from oxygen that can damage lipids, proteins and DNA. They can also act as cell signaling molecules. ROS are produced every time at every single moment in every aerobic organism by the respiratory electron transport chain at the mitochondria, p450 cytochrome reactions, peroxisomal fatty acid metabolism and NADPH oxidase activity, but, normally, there is a balance between ROS and antioxidant molecules. It is when this balance is broken when ROS begin their harmful activity and an oxidative stress situation is established (Borges et al, 2007).

In mild and chronic periodontitis, ROS generation is enhanced and plasma antioxidant levels are depleted. This ROS generation at the site of periodontitis can also have systemic effects on other organs as ROS can diffuse into the blood stream and reach other places on the organism. This is specially worth investigating on it, because it is known that serious diseases as cardiovascular disease and diabetes are related with ROS generation at the organism.

People suffering from chronic periodontitis have higher $C$ reactive protein (CRP) plasma levels. This is a powerful pro inflammatory molecule that also participates in other pathologies as obesity (D'Aiuto et al, 2010) and it is a good marker of the development of atherosclerosis and myocardial infarction (Tonetti, 2009).

As It has mentioned before, periodontitis appears as the result of all the cytokines, ROS and inflammatory molecules released from the microorganisms and the host immune cells at the site of the inflammation at the teeth. The presence of a high amount of neutrophils, as this is the main host response to bacterial invasions, give us the idea of the great amount of ROS that can be released from them to combat the infection. There is also systemic inflammation in periodontitis as assessed in some studies measuring plasma CRP (Paraskevas et al, 2008), which can influence the production or ROS all over the organism.

The most important ROS involved in periodontitis are:

Hydroxyl radical $\left({ }^{*} \mathrm{OH}\right)$, very active in damaging DNA proteins and lipids. It is able to initiate the lipid peroxidation chain, leading to vasodilation and bone reabsorption. Its molecular mechanism of action is by stimulation of the NF-K B-IKB complex, activating then $\mathrm{NK}-\mathrm{KB}$, which helps nuclear translocation and downstream of proinflammatory cytokines as IL-2, IL-6, IL-8, $\beta$-interferon and TNF-a (Borges et al, 2007).

Hydrogen peroxide $\left(\mathrm{H}_{2} \mathrm{O}_{2}\right)$, capable of crossing the nuclear membrane and damaging the DNA. DNA damage is measured by quantifying levels of 8-hydroxydeoxyguanosine (8OHDG), that is increased in periodontal tissues in periodontitis (Ekundi et al, 2010).

Superoxide anion $\left(\mathrm{O}_{2}{ }^{{ }^{*}}\right)$, involved in bone reabsorption as many studies have corroborated by measuring the presence of this anion near the sites of bone reabsorption. 
The most important cellular antioxidants are the enzymes superoxide dismutase (SOD), catalase, and glutathione peroxidase, glutathione-S transferases and aldehyde dehydrogenases. There are also non enzymatic antioxidants as carotenoids, vitamin E, C, coenzyme $\mathrm{Q}_{10}$, a-lipoic acid, antioxidant minerals (selenium, zinc, copper, manganese), phenols, flavonoids, lycopene or hydroxytyrosol. When levels of these enzymes are lowered, the organism is unable to neutralize ROS and they can exert its harmful effect (Vicents \& Taylor, 2006).

So, ROS are involved both in tissue destruction in periodontitis and in systemic inflammation. They are also involved in several systemic diseases as metabolic syndrome, diabetes, obesity or cardiovascular disease. This means that it can be a link between periodontitis and these diseases, which altogether are risk factors for cardiovascular disease. We are going to inquire in the relation of ROS, oxidative stress and these diseases to understand why the generation of ROS in periodontitis can be linked to the apparition or deterioration of these diseases that in the end lead to cardiovascular disease.

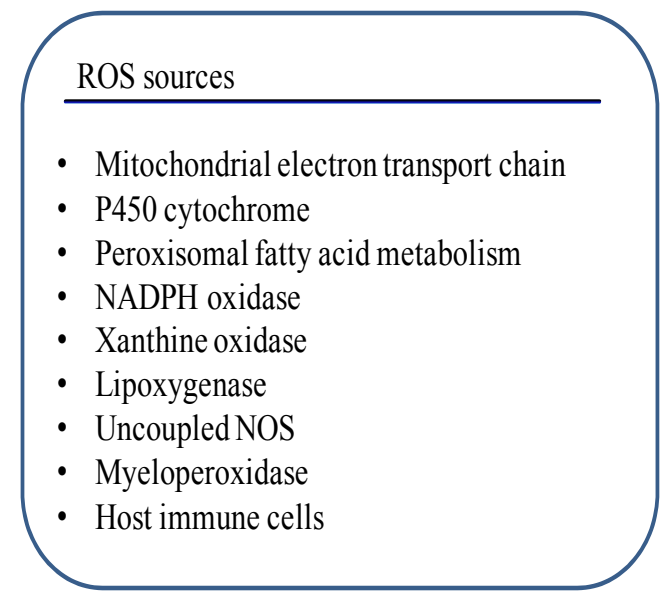

Fig. 1. ROS sources.

\section{Cardiovascular disease}

Cardiovascular diseases are the world's largest killers, claiming 17.1 million lives a year, representing 29\% of all global deaths. Cardiovascular diseases (CVDs) are a group of disorders of the heart and blood vessels and include:

- Coronary heart disease

- Cerebrovascular disease

- Peripheral arterial disease

- Rheumatic heart disease

- Congenital heart disease

- Deep vein thrombosis and pulmonary embolism

Heart attacks and strokes are usually acute events and are mainly caused by a blockage that prevents blood from flowing to the heart or brain. The most common reason for this is a 
build-up of fatty deposits on the inner walls of the blood vessels that supply the heart or brain. Strokes can also be caused by bleeding from a blood vessel in the brain or from blood clots.

\subsection{Cardiovascular disease and ROS}

Although hypertension is the main cause for having a cardiovascular event, ROS can also affect and damage cardiac tissues directly, leaving heart more susceptible to any other damage. Sources of ROS in cardiac tissue are not already unknown for us. These sources are the mitochondrial respiratory chain, NADPH oxidases, xanthine oxidases, lipoxygenase, uncoupled NOS and myeloperoxidase. There are evidences that ROS production by these systems are involved in cardiac damage and the apparition of heart failure diseases as congestive heart failure, angiotensin II dependent cardiac hypertrophy or cardiac fibrosis ,inter alia.

It is known that ROS can harm cardiac tissue because after reperfusion injury, there is a great increase in ROS production, attributed to an overload of all the ROS sources mentioned above and to a high infiltration of neutrophils that release pro inflammatory citokynes. This will damage myocytes, impair contractile function and contribute to capillary leakage (Chang et al, 2010).

\subsection{Cardiovascular disease and periodontitis}

Nowadays, there are several studies linking periodontitis with cardiovascular diseases (Paquette et al, 2007). We have seen how periodontitis can lead to an oxidative stress status in the organisms that will promote the onset of other diseases as obesity, diabetes or hypertension that are risk factors for cardiovascular disease and the possible mechanisms of how this happens. There is also evidence of direct damage by ROS to cardiac tissue and even DNA from periodontal pathogens in atheroma plaques, suggesting that they can also spread from the periodontium and travel throughout the blood stream (Tonetti, 2009). But there is another one important fact about how periodontitis can affect cardiovascular health, and it is the formation of the atheroma plaque or atherosclerosis.

Atherosclerosis begins with stimuli from LDL or pro inflammatory proteins to endothelial cells which will express adhesion factors, chemokines and growth factors that attract monocytes from circulating blood. These monocytes can enter the intima and differentiate into macrophages. Macrophages then are able to uptake oxidized LDL and cholesterol and they transform in foam cells, damaging the intima. Then, activation of $\mathrm{T}$ lymphocytes, smooth muscle cells proliferation and migration and extracellular matrix deposition interact with molecules in the intima, promoting necrosis and forming fibrous plaques. These plaques are atheroma plaques and will grow and enlarge. Consequences of this could be occlusion of the artery where the atheroma plaque is formed or rupture of the plaque, releasing a plaque fragment that may be transformed into a thrombus that can obstruct blood flow anywhere on the body, with the risk of producing an acute cardiovascular event or stroke (Chang et al, 2010). ROS periodontitis production could be dangerous due to the oxidation of LDL and its pro atherogenic role. People with periodontitis have higher levels of plasma oxidized LDL levels, which means higher risk of developing atheroma plaque. Treatments directed to improve periodontitis achieved a lowering in oxidized LDL plasma 
levels (Tamaki et al, 2010). Platelets also contribute greatly to atheroma plaque formation. It is known that platelet activation is increased in people suffering from periodontitis, being greater this activation as periodontitis severity increases (Papapanagiotou et al, 2009). Furthermore, platelets are able to bind fibrinogen and form thrombi that can occlude any blood vessel by themselves without the need of an atheroma plaque. The reason of this platelet activation could be in the action of periodontitis associated bacteria. It is to say that platelets are activated by the vasodilator-stimulated phosphoprotein (VASP) whose mission is to regulate platelet activation. When phosphorylated, VASP inhibit platelet activation, but in periodontitis, there is a decrease in VASP phosphorylation due to direct interaction between these periodontitis associated bacteria and VASP, leading to higher platelet activation (Laky et al, 2011).

But atherogenesis is not the only risk factor involved in cardiovascular events. Mitochondria play an important part in heart damage. Mitochondria are the primary source of intracellular ROS. When damaged, mitochondria suffer a dysfunction in their activity making them to produce huge amounts of ROS and release pro apoptotic proteins into the cytosol that can trigger apoptosis in the cell. ROS are able to slowly damage mitochondria but the key is the damage to mitochondrial DNA (mtDNA) that is produced when, because of punctual mutations in mtDNA due to ROS action, mitochondria is unable to properly work and begins producing high amounts of ROS and these pro apoptotic proteins as cytochrome $\mathrm{c}$ or apoptosis inducing factor (AIF) that destroy the cell. People suffering from atherosclerosis have higher mtDNA damage (Humphrey et al, 2008).

High chain long fatty acids (FA) plasma concentration is also a common link between periodontitis, diabetes and cardiovascular risk. FA plasma concentrations are elevated in periodontitis (Ramirez-Tortosa et al, 2010) as they are in diabetes (Liu et al, 2010). Both elevations can together bring up a very high plasma fatty acids concentration. Normally, heart uses fatty acids as energy source generated by $\beta$-oxidation and also uses glucose in a lesser amount. This high plasma fatty acid concentration will force the heart to use almost only free fatty acids as substrate for energy production. Fatty acids get into the cell by passive diffusion ( $20 \%$ of fatty acids) and by active transport mediated by CD36 transporter (80\% of fatty acids). Once inside the cell, fatty acids bind to a cytosolic fatty acid-binding protein and are transported to the outer mitochondrial membrane. There, fatty acids are sterified and get inside the mitochondrial matrix, where $\beta$-oxidation is performed. When the FA concentration is excessive, glycolytic intermediates and intracellular lipids accumulate because mitochondria are unable to $\beta$-oxidate all the FA. This FA excess will produce ROS inside the cardiomyocyte cytoplasm as a result of an increased FA oxidation. There will also be accumulation of DAG and ceramides. ROS and DAG will activate JNK, IKK kinases and PKC respectively. Together, they will downregulate insulin action through serine phosphorilation of insulin receptor substrate-1 (IRS-1), what will inactivate IRS-1 and stop the insulin pathway. Also, ceramides will inhibit Akt, what will also neutralize insulin action. It is still unknown why, but diabetes leads to heart hypertrophy and failure independently of atherosclerosis or hypertension. Experiments with rodents that lacked CD36 transporter showed that those rodents did not have any cardiac dysfunction. This could mean that an excessive FA intake and intramyocardial should be involved in cardiac failure somehow. A possible reason is the generation of ROS inside cardiomyocytes. 
As it is known, ROS can activate NF- $\mathrm{k} \beta$ with its consequent production of growth factors and pro inflammatory cytokines. ROS can also activate matrix metalloproteinases that have the capacity of remodeling the extracellular matrix and cleave sarcomeric proteins such as troponin-1 and myosin light chains-1 (MLC-1) what will cause deficiencies in heart contractility and even apoptosis if damage is too high. As important is the role of heart mitochondria in this process. ROS are able to damage mitochondria and make it produce even more ROS and loss of functionality. Damage to mitochondria will release pro apoptotic proteins such as cytochrome c, caspases 3 and 9 that will lead to cell death. If mitochrondrion is damaged and there is no apoptosis, loss of function will reduce $\beta$ oxidation rate and make FA accumulate, producing even more ROS and beginning the cycle (Dirkx et al, 2011).

Mitochondria have a key role in cardiac failure and remodeling. ROS generation is increased in failing myocardium as demonstrated by measurement of 8-iso-prostaglandin F2a. Heart is the main oxygen user in the body so ROS generation rate is higher in heart than in other organs. When heart is damaged, heart mitochondria ROS generation is enhanced, being able to damage myocytes, although, also NADPH oxidase and xanthine oxidase are able to produce ROS in heart and damage myocytes. Mitochondria have their own DNA (mtDNA), more susceptible to damage because:

1. It is less protected than nuclear DNA that possesses histones.

2. mtDNA is located in the mitochondrial matrix, where ROS production is constant by the electron transport chain activity.

3. mtDNA repair is not so effective as nuclear DNA repair.

There is also cardiac remodeling by activation of metalloproteinases by ROS. Cardiac metalloproteinases activity is increased in failing hearts possibly due to the high ROS generation in damaged cardiac tissue. Studies with metalloproteinases inhibitors show that inhibition limits early left ventricular dilatation in murine models (Tsutsui et al, 2009).

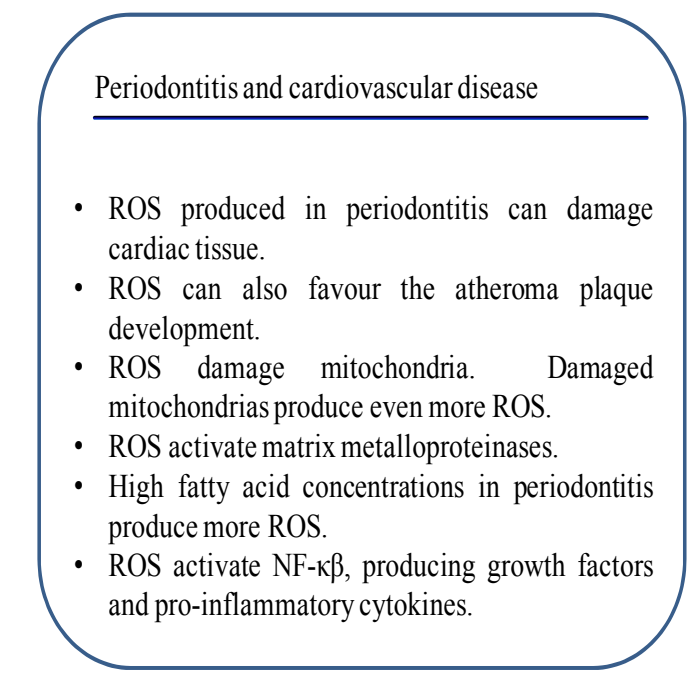

Fig. 2. Periodontitis and cardiovascular disease 


\section{Metalloproteinases functions}

- Participate in normal tissue remodelling.

- Regulate cell migration, invasion, proliferation and apoptosis.

- Regulate branching morphogenesis.

- Participate in angiogenesis.

- Participate in wound healing.

- Regulate extracelular matrix degradation.

Fig. 3. Metalloproteinases functions

Mitochondria and periodontitis are also well related. Bacterial lipopolysaccharide released during periodontitis by bacteria is able to damage mitochondria by decreasing mitochondria membrane potential, mitochondrial mass, $\mathrm{CoQ}_{10}$, and protein expression. $\mathrm{CoQ}_{10}$ deficiency also makes a decrease in complex II + III, III and IV activities. This taken together raises ROS production inside the mitochondria. The next step before ROS damage is produced is liberation of cytochrome c, procaspase-9, caspase-3 and endonuclease G, resulting in DNA degradation and cell death.

\section{Metabolic syndrome}

Metabolic syndrome is a compound of afflictions that comprises hypertension, diabetes and dyslipidemia caused by abnormal obesity due to physical inactivity and overeating. According to the American Heart Association, metabolic syndrome comprises several risk factors as:

- Abdominal obesity (excessive fat tissue in and around the abdomen).

- Atherogenic dyslipidemia (blood fat disorders - high triglycerides, low high density lipoprotein (HDL) cholesterol and high low density lipoprotein (LDL) cholesterol that foster plaque buildups in artery walls).

- Elevated blood pressure.

- Insulin resistance or glucose intolerance.

- Prothrombotic state (e.g., high fibrinogen or plasminogen activator inhibitor-1 in the blood).

- $\quad$ Proinflammatory state (e.g., elevated C-reactive protein in the blood).

Metabolic syndrome is associated with periodontitis as shown on epidemiological studies that demonstrate the relationship. In a group of 1315 patients, the prevalence of metabolic syndrome was higher in patients with advanced periodontitis $(66.7 \%)$ than in those periodontally healthy $(48.8 \%$ )(Borges et al, 2007). Another study from 13,710 participants in the NHANES III (Third National Health and Nutrition Examination Survey) showed a direct relationship between periodontitis and the prevalence of metabolic syndrome $(37 \%$ in those with severe periodontitis vs. $18 \%$ in those with mild or no periodontitis) (D'Aiuto et al, 2008; Bullon et al, 2009). 
People with the metabolic syndrome are at increased risk of coronary heart disease and other diseases related to plaque buildups in artery walls (e.g., stroke and peripheral vascular disease) and type II diabetes.

Abdominal obesity, insulin resistance and hypertension seem to be the most important of these risk factors in order to develop a cardiovascular disease. They are also influenced by oxidative stress as it will be described later.This syndrome is clinically important because of the associated cardiovascular risk accumulation, which exceeds that of the component parts. Thus, it is important to elucidate the pathophysiology of metabolic syndrome to prevent the development of the associated cardiovascular disease (CVD).

\section{Obesity}

Obesity is a state of fat mass excess. Adipose tissue is formed by adipose cells that store lipids and also preadipocites. Adipocites accumulate lipids and grow in number and shape. There is also a high number of infiltrating macrophages due to the expression of interleukins and a high rate of preadipocites differentiation into macrophages by peroxisome proliferator-activated receptor gamma (PPAR- $\gamma$ ) and TNF-a.

Adipocites release also many other molecules that have influence on other biological processes, for example, adiponectin, which favors insulin sensibility, lipid oxidation and has vascular protective properties but is downregulated in obesity, or angiotensinogen, that acts over blood pressure. This molecule concentration is reduced in metabolic syndrome and in diabetes.

\subsection{Obesity, periodontitis and oxidative stress}

Obesity is one of the most important parts of metabolic syndrome. It is known that there is systemic chronic inflammation and increased ROS generation in obesity, thus, leading to an oxidative stress situation. This is mainly due to the production of TNF-a, IL-6, non-esterified fatty acids, angiotensinogen and CRP (Ando \& Fujita, 2009). Possible reasons why there is an increased ROS production in obesity can be: hyperglycemia, increased muscle activity to carry excessive weight, elevated tissue lipid levels, inadequate antioxidant defenses, chronic inflammation, endothelial ROS production and hyperleptinemia (D'Aiuto et al, 2008). We can see that many of these factors as hyperglycemia, inadequate antioxidant defenses, chronic inflammation and endothelial ROS production depends on other pathologies related to metabolic syndrome, and can be worsened if these pathologies are in an advance state of the disease, as diabetes or atherosclerosis.

Hyperglycemia and obesity are heavily linked, and therefore, obesity and diabetes. Obesity induces insulin resistance, suppress the expression of insulin receptors in fat tissue, causing glucose deregulation, hyperglycemia and later, $\beta$-cell destruction in pancreas, promoting diabetes. Due to the impossibility to catch glucose from cells because of this insulin resistance, glucose accumulates in the blood stream, leading to hyperglycemia. This leads to 3 different pathways that will end with the production of ROS:

- The first pathway is the polyol pathway where glucose is converted to sorbitol, which in excess produces oxidative damage. This pathway includes the participation of 
nicotinamide adenine dinucleotide phosphate (NADPH) oxidase activity, that produces $\mathrm{O}_{2}{ }^{*}$, especially in the endothelium.

- The second pathway is the forming of advanced glycosylation end products (AGE) because of the reaction between glucose and proteins, lipids and nucleic acids. This AGEs binds to specific cell surface receptors (RAGE) and this promote the production of NF- $\mathrm{k} \beta$, which in turn activates PKC, sorbitol and transcription of vascular cell adhesion molecule-1 (VCAM-1) and intracellular adhesion molecule-1 (ICAM-1). All this molecules activation will raise ROS levels.

- The third pathway is just the glucose auto-oxidation with the generation of oxidants with similar reactivity as $\mathrm{OH}^{*}$ and $\mathrm{O}_{2}{ }^{*}$ - radicals.

All this three pathways together with an increased metabolic ROS production in obese people, inadequate antioxidant defenses due to an unsuitable diet and continuous antioxidant attrition will bring up an oxidative stress situation.

\subsection{Obesity, periodontitis and fatty acids}

It is important to talk about the lipid profile and changes in lipid peroxidation with ROS production in obesity because it could be linked with periodontitis and cardiovascular risk as Ramirez-Tortosa et al.(2010) found in one of their studies. Fat storage, excessive blood lipids and dyslipidemia are found in obesity with an increase in plasma saturated, monounsaturated and polyunsaturated fatty acids. Periodontitis is a systemic inflammation and ROS generator, what will contribute to the oxidation of LDL that are also elevated in obesity.

Dietary intake of lipids can also modify plasma lipid profile as people with high intake of saturated and n-6 polyunsaturated fatty acids, but not those with a higher intake of n-3 polyunsaturated fatty acids, have increased cardiovascular risk due to an increased oxidized lipoproteins and vascular inflammation. Total n-3 polyunsaturated fatty acids were associated with lower levels of pro inflammatory markers (IL-6, TNF- $a$, CRP), higher levels of anti-inflammatory markers (soluble IL-6r, IL-10, TGF-a) and lower levels of some markers of endothelial activation (sVCAM-1 and sICAM-1). This means that n-3 polyunsaturated fatty acids are cardio protective, but in periodontitis patients, n- 6 polyunsaturated and saturated fatty acids predominate in plasma, a proatherogenic lipid profile that is responsible for the production of high levels of pro inflammatory TNF-a and proatherogenic sVCAM-1 and oxidized LDL (Ramirez-Tortosa et al, 2010).

There are many ways in which lipids can contribute to oxidative stress as uncoupling of mitochondria, susceptibility to ROS attack and production of PKC that also raise glucose levels. Also NADPH oxidase activity is increased in adipose tissue and there is a reduced activity of antioxidant enzymes as superoxide dismutase (SOD). All these reactions generate ROS, leading to lipid and protein oxidation and finally to oxidative stress.

As seen on this study, there are several changes in the lipid profile in common between periodontitis and obesity. These changes were in cholesterol, triacylglycerols, LDL, and very low density lipoproteins (VLDL) plasma levels that increased both in periodontitis groups as shown in this study and in obesity patients while HDL levels were decreased. As periodontitis and obesity are two diseases that share in common general systemic low grade 
inflammation, IL-6 is elevated in both diseases. One of IL-6 effects is to decrease lipoprotein lipase activity, a key enzyme involved in triglycerides catabolism and formation of adipose tissue. Reduction in its activity will produce hypertriglyceridemia (Monteiro et al, 2009).

While ROS have adverse effects on obesity development, and its production is increased in obesity, periodontitis ROS production may increment drastically ROS levels, worsening the oxidative stress situation in obese patients with periodontitis. Lots of studies link the relationship between obesity and oxidative stress (Vincent \& Taylor, 2006) by measuring lipid peroxidation biomarkers as malondialdehyde (MDA), thiobarbituric reactive acid substances (TBARS), $\mathrm{C}$ reactive protein (CRP), lipid hydroperoxides, conjugated dienes, 4hidroxynonenal $(4 \mathrm{HNE})$ and $\mathrm{F}_{2}$ isoprostanes (8-epiPGF $2 \mathrm{a}$ ). Those studies showed an increase in these biomarker levels in obese patients and also a decrease in antioxidant enzymes, showing that there is an increased oxidative stress in this situation. Plasma MDA levels are increased in obese patients and it is involved in systemic oxidative stress and in impairment of normal glucose metabolism in obese people, which will also contribute to the instauration of diabetes, another important risk factor in metabolic syndrome for the development of cardiovascular diseases. But not also MDA levels are increased in obesity. All the other obesity biomarkers mentioned above were increased.

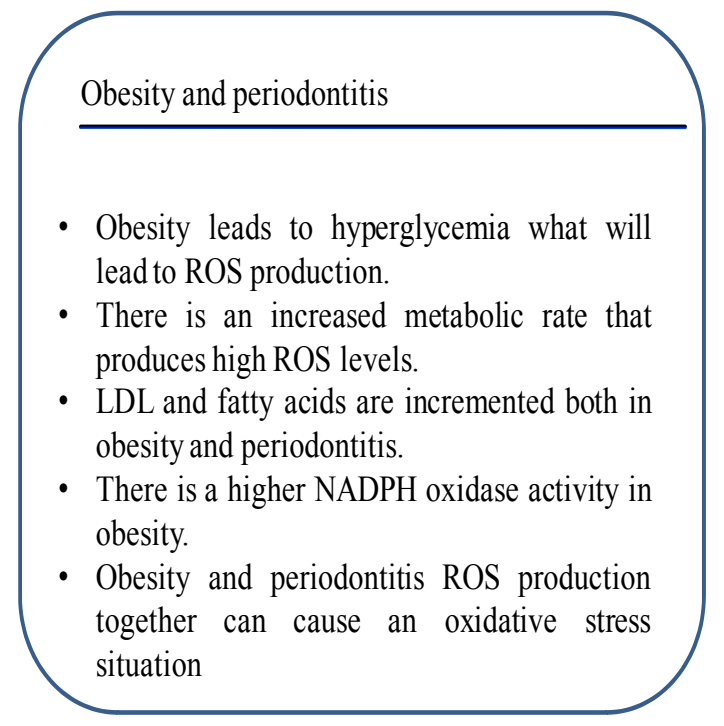

Fig. 4. Obesity and periodontitis

It is evident the relation between the plasma lipid profile and the risk of developing atherogenesis. Specially, a high LDL concentration may lead to the development of the atherogenic plaque and subsequently raise the level of risk of suffering cardiovascular diseases. Now, there are evidences of an increase in these levels of plasma LDL in periodontitis (Ramirez-Tortosa et al, 2010). If we sum up this phenomenon with the rise of plasma LDL happening in obesity, we probably will have a high rise in plasma oxidized LDL and therefore a higher risk in developing atheroma plaque. 
Another factor that promotes oxidative stress situations in obesity is the level of plasma leptin. Leptin is a polypeptide hormone that regulates food intake acting on hypothalamic centers. It is produced by adipose tissue. It is a cardiovascular risk factor in obese people. Leptin is chemically similar to IL-6 cytokine family and so it has pro inflammatory properties stimulating the proliferation of monocytes and macrophages and inflammatory cytokines. So, indirectly, it stimulates NADPH oxidase activity. It also stimulates directly $\mathrm{H}_{2} \mathrm{O}_{2}$ and $\mathrm{OH}^{*}$ and reduces the activity of paranoxase (PON-1), and antioxidant enzyme and this reduction increases urinary and plasma 8-isoprostane and plasma MDA and hydroperoxides. Because PON-1 is involved in preventing the accumulation of peroxides in LDL, reduction in the activity of this enzyme could contribute to CAD development.

\section{Obesity, periodontitis and ROS}

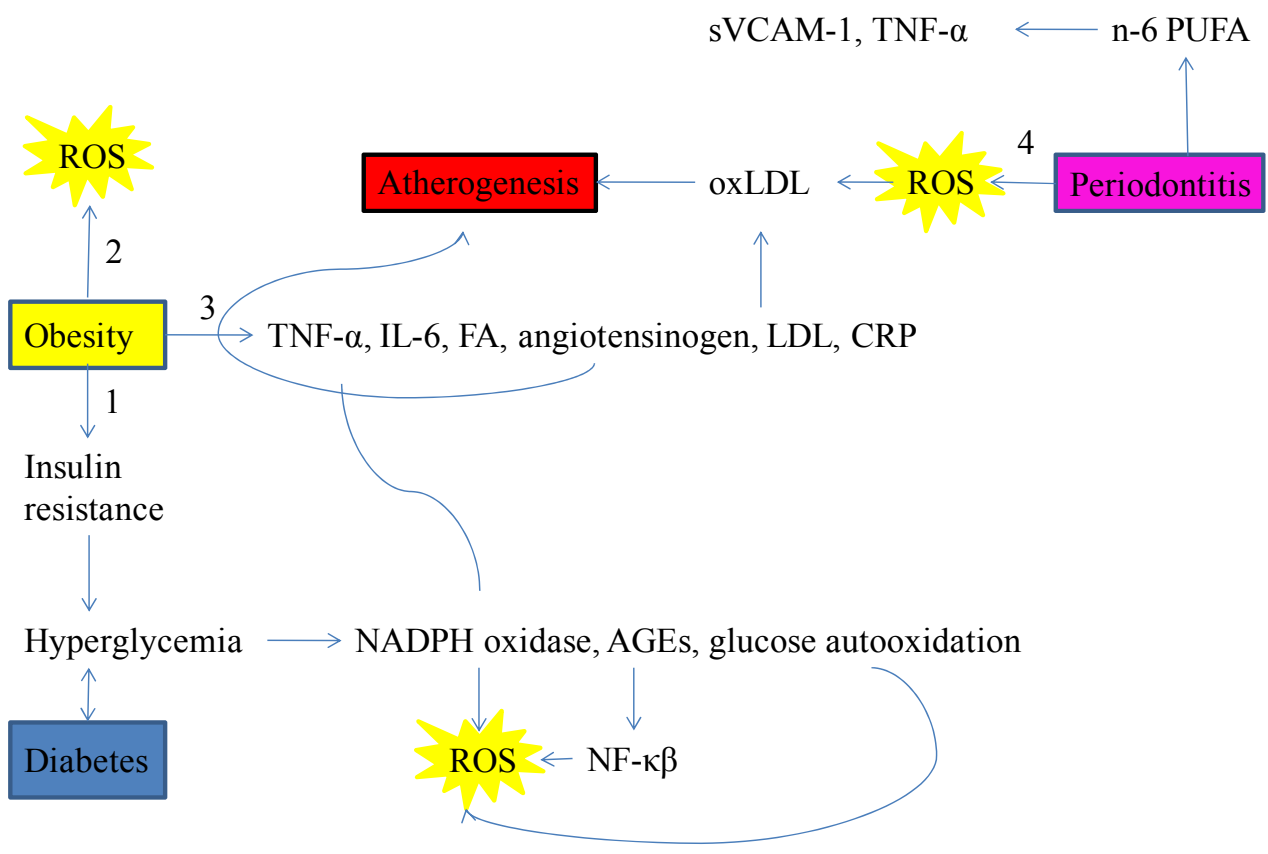

Fig. 5. Obesity, periodontitis and ROS. 1). Obesity is able to produce insulin resistance that will lead to hyperglycemia and diabetes. This hyperglycemia rises NADPH oxidase activity, favors the formation of AGEs and provoke glucose autooxidation. These three processes will generate ROS. 2). There is also a ROS generation by obesity due to a higher metabolism in obesity that will sum up to the produced by hyperglycemia. 3). There is an increased LDL and angiotensinogen plasma concentration in obesity that will support the formation of the atheroma plaque. 4). In addition, periodontitis is able to produce even more ROS to the organism, worsening the situation and leading to an oxidative stress status. 
The last cardiovascular risk factor that obesity can modify is the endothelial function. Obese people also suffer from hypertension. Vascular endothelium presents some ROS sources that are deregulated in obesity because of the imbalance in rennin-angiotensin system as we saw before. Angiotensinogen converts to angiotensin II, leading to NADPH oxidase activity and xanthine oxidoreductase activity increase, LDL uptake by macrophages, nitric oxide synthase enzymatic uncoupling and the consequent formation of ROS, antioxidant depletion, lipid peroxidation and oxidative stress situation (Vicent \& Taylor, 2006). Obesity is a risk factor for the development of glucose resistance, hypertension, diabetes, possibly due to the production of ROS and high plasma lipid levels.

\section{Insulin resistance, glucose impairment, diabetes and periodontitis}

Diabetes mellitus is a syndrome where carbohydrates, lipids and proteins metabolism is altered due to a lack of insulin secretion or to a decrease in tissues response to insulin. There are two kinds of diabetes:

- Diabetes mellitus type I: also called insulin dependent diabetes mellitus. In this diabetes, there is no production of insulin by pancreas.

- Diabetes mellitus type II: also called no insulin dependent diabetes mellitus. This diabetes presents a decrease in tissues response to insulin called insulin resistance.

The consequences of diabetes is that every cell, except encephalic cells, will not be able to properly use insulin, so plasma glucose levels raise and appears hyperglycemia.

Type II diabetes is strongly related to obesity. Almost every obese develop type II diabetes. The link between obesity and diabetes was unclear till the recent theory of ROS. As we saw before, obesity is an important factor in the development of diabetes because of the release of pro inflammatory cytokines and production of ROS that lead to an oxidative stress situation and the insulin resistance situation in obesity.

People with diabetes are at high risk of developing other health problems as macroangiopathies and microangiopathies. The first one consists on an accelerated atheromatosis that will have damaging effects over cardiovascular health. Microangiopathies affects to retina, kidney and peripheral nerves. Affliction of the kidney is an important fact in the instauration of a renal failure and diabetes is the first cause of chronic renal insufficiency. So, diabetes can cause accelerated atheromatosis with hypertension and chronic renal insufficiency, leaving the patient to a high cardiovascular disease risk situation.

Hyperglycemia produce decreased activity of vasodilators such as nitric oxide (NO), increased activity of angiotensin II, endothelin-1 and favors the production of permeability factors as vascular endothelial growth factor (VEGF). Later on, there is microvascular cell loss and capillary occlusion due to extracellular matrix overproduction by action of growth factors such as TGF- $\beta$ and to deposition of extravasated acid-Schiff-positive plasma proteins. With respect to hypertension, these changes will produce glomerulosclerosis in the kidney, which will cause renal malfunction leading to hypertension.

Insulin resistance and hyperglycemia also provokes changes in blood lipid profile. Cholesterol enriched apolipoprotein B containing remnant particles levels, which is proatherogenic, is elevated in diabetes. 
In diabetes, endothelial cells produce an excess of AGEs. Intracellular hyperglycemia originates autooxidation of glucose that ends with the formation of dicarbonyls that reacts with amino groups of intracellular and extracellular proteins to form AGEs. This AGEs importance keeps on the fact that, inter alia, they are able to react with macrophages and mesangial cells AGE receptors and to produce intracellular ROS that activates NF-k $\beta$ pathway with its consequent production of growth factors and pro inflammatory cytokines. AGE can also be formed on collagen to form very stable collagen macromolecules, resistant to normal enzymatic degradation. This AGE-modified collagen accumulates in the walls of blood vessels, narrowing the lumen. It also has affinity for LDL, so, AGE-modified collagen scavenges LDL and deposits them in the blood vessels walls, contributing to the formation of atheroma plaque and further cardiovascular problems. AGE-modified collagen has been shown to be found also in periodontal blood vessels.

AGE also promote the formation of VEGF that induces neovascularization and plays a major role in microvascular diabetes complications (Mealey \& Oates, 2006). Another important molecule upregulated in diabetes is protein kinase $C$ (PKC). This enzyme is activated by diacylglycerol, which levels are elevated in diabetes. PKC has several functions:

- Decrease eNOS activity and increase endothelin-1 activity, producing blood flow abnormalities.

- Increase VEGF levels, promoting vascular permeability and angiongenesis.

- Induce expression of TGF- $\beta$ which promotes capillary occlusion.

- Induce overexpression of the fibrinolytic inhibitor PAI-1. By doing this, vascular occlusion is promoted.

- $\quad$ Activates NF-k $\beta$ pathway, releasing pro inflammatory cytokines.

- Activate NADPH oxidases that release ROS, leading to multiple effects in the organism.

Together with the formation of AGE and activation of PKC, in diabetes there is an increased flux in the polyol pathway and in the hexosamine pathway, both of them contributes to the progression of diabetes. But these four processes link together in a common pathway, the overproduction of superoxide by mitochondrial electron transport chain.

There is a value in the inner mitochondrial membrane proton gradient that when exceeded, superoxide production is highly increased. This limit is reached in diabetes, because of the overproduction of electron donors by the tricarboxilic acid cycle due to hyperglycemia. This is shown by measures of ROS that are elevated in mitochondria in diabetic patients. Overexpression of manganese superoxide dismutase (MnSOD) lowered these ROS levels and also lowered all the four factors that contribute to diabetes progression: formation of AGE, PCK, increased flux in polyol pathway and in hexosamine pathway. By inhibiting mitochondrial superoxide production, activation of NF-k $\beta$ is also inhibited. But not only MnSOD inhibits these processes, it also suppresses the increase in collagen synthesis in mesangial cells, decreases hyperglycemia induced apoptosis in dorsal root ganglion neurons, blocks hyperglycemia induced monocyte adhesion, prevents the inhibition of the anti atherogenic prostacyclin synthetase, PPAR $-\gamma$ and endothelial nitric oxide synthase (eNOS).

This gives us an idea of how important is mitochondrial superoxide production and mitochondrial integrity in the development and progression of this disease. All damage 
done to mitochondria could affect its standard ROS production and transform the mitochondria in a potent ROS generator. This will not only affect progression of diabetes but also obesity, inflammation, hypertension, periodontitis, generating an oxidative stress situation (Brownlee, 2001).

\subsection{Diabetes, periodontitis and oxidative stress}

Diabetes and periodontitis are strongly related diseases. People suffering from diabetes have higher risk of developing periodontitis and people suffering from periodontitis have higher risk of developing diabetes. It is a positive feedback. Treatment of periodontal disease improves some diabetes complications as hyperglycemia or glycated hemoglobin. The way diabetes affects periodontitis may be as the result of the above mentioned processes that leads to release of pro inflammatory cytokines, activation of PKC that activates NADPH oxidase, accumulation of AGEs and deficiencies in tissue healing in diabetes. This would ease the damage produced in the periodontium and is associated with chronic periodontitis. Hyperglycemia produces inhibition of osteoblastic cell proliferation and collagen production so bone regeneration is attenuated and damage produced in the periodontium is this way higher, having mechanical diminished bone properties in the new formed teeth.

Studies have shown that specially TNF-a, IL- 6 and IL-1 $\beta$ are markedly elevated in diabetes. Macrophages from diabetic patients release more TNF- $\mathrm{a}, \mathrm{PKC}$ and $\mathrm{O}_{2}{ }^{-}$than macrophages from healthy patients (Karima et al, 2005), possibly due to a high glucose level and oxidative stress. Both diseases, periodontitis and diabetes are able to activate an immune host response with release of pro inflammatory cytokines and instauration of an oxidative stress situation. When both join together in the same patient, they can act synergistically (Nassar et al, 2007).

ROS can also be a final step in which some pathways converge to produce insulin resistance. It has been shown that TNF-a and dexamethasone are able to produce ROS. Although TNF$\alpha$ is pro inflammatory and dexamethasone is an anti-inflammatory agent, both raise ROS levels and produce insulin resistance. Treatment with antioxidant molecules is able to decrease insulin resistance, measured as the defect in insulin mediated glucose uptake. Also, transgenic models of cell lines genetically modified to overexpress antioxidant enzymes and transgenic obese mice treated with antioxidants prevented insulin resistance after treatment with TNF-a and dexamethasone. This is indicative of the involvement of ROS in insulin resistance as antioxidant agents can prevent this process. Other evidences of ROS implicated in insulin resistance are the fact that in obesity, ROS are increased due to a constant inflammatory state, and this produce insulin resistance leading to diabetes. Also adipocytes treated with high doses of $\mathrm{H}_{2} \mathrm{O}_{2}$ or ROS inducers produce insulin resistance. The possible mechanism of ROS affecting insulin resistance can depend on the magnitude of ROS production, cell type affected, time of exposure, specific type of ROS or other factors that will activate any of the involved pathways as FoxO, MAPK, JAK/STAT, p53, phospholipase $\mathrm{C}, \mathrm{PI}(3) \mathrm{K}$ and other proteins encoded genes. A possible way is through activation of JNK by TNF-a. Antioxidant treatment reduces JNK insulin resistance mediated activity (Houstin et al, 2006). This could be the reason of how periodontitis could affect or worsen insulin 
resistance, by production of ROS that in the long term will produce diabetes as similarly happens in obesity. In periodontitis patients with type II diabetes, there is an increased C reactive protein level, which means there is increased IL-6 concentration that could exacerbate insulin resistance and contribute to diabetes worsening (Aspriello et al, 2011; Gomes-Filho et al, 2011).

It is important to have a good control of these diseases, preventing and if necessary, treating them. This is because as the worst the control of diabetes is the worst the effects on periodontitis severity and ROS production will be (Tsai et al, 2002; Lalla \& Papapanou, 2011). As higher glycemic status, higher activity of PKC, NADPH oxidase and levels of DAG. DAG increases with hyperglycemia through the glycolytic/glycerol-3-phosphate acyltransferase pathway. This increase in DAG triggers activation of PKC. Also IL- $1 \beta$ levels are double in patients with poor glycemic control ( $>8 \%$ glycohemoglobin test HbA1c) than in patients with good glycemic control. Periodontal treatment is able to reduce systemic inflammation, reduce serum TNF-a, C-reactive protein, IL-6, fibrinogen concentration, improves HbA1c levels and raise adiponectin concentration (Lalla and Papapanou, 2011).

Disease control consists on medication, healthy lifestyle and weight control for diabetes and proper oral hygiene for periodontitis. Avoid smoking for both diseases. It has been demonstrated that clinically treating periodontitis can reduce up to $48 \%$ IL-6 levels in patients with periodontitis and type II diabetes (Tamaki et al, 2010) and also can reduce CRP levels (Marcaccini et al, 2009).

Diabetes is strongly related with hypertension and thus, cardiovascular disease. As mentioned above, one of the major complications of diabetes is the apparition of macroangiopathies and microangiopathies. These processes are favored by AGE products formed as a result of hyperglycemia and ROS production, protein kinase $C$ and the renin angiotensin system activation.

AGEs products promote microangiopathies as seen by its apoptotic action over mesangial cells and the promotion of vascular endothelial growth factor (VEGF) which will contribute to glomerular hyperfiltration, an early renal dysfunction. Also, AGEs stimulate the production of insulin like growth factor-I, -II, PDGF and TGF- $\beta$ in mesangial cells, which in turn produce type IV collagen, laminin and fibronectin. TGF- $\beta$ is an important molecule that plays an important role in the pathogenesis of glomerulosclerosis and tubulointerstitial fibrosis. AGE-RAGE system is also related with the renin angiotensin system because angiotensin II produce ROS in renal cells and ROS are causative of AGEs. Then AGEs activate mesangial TGF- $\beta$-Smad system which activates production of angiotensin II (Yamagishi et al, 2011).

There are several ways for AGEs products to contribute to the development of macroangiopathies such as decrease in the elasticity of the vasculature, quenching of $\mathrm{NO}$, decreasing vasodilation, increased oxidative stress that impairs NO synthase and produces peroxinitrite (ONOO-), increase in plasma LDL or activation of atherosclerosis related genes by ROS generation as ICAM-1, VCAM-1, monocyte chemotactic protein-1(MCP-1), plasminogen activator inhibitor-1 (PAI-1), tissue factor, VEGF and RAGE. There is also a deficient tissue repair situation in diabetes because of a decrease in endothelial progenitor cells (EPCs) number, function and mobilization. These cells are capable of differentiate in 
endothelial cells. Deficit in endothelial repair may lead to accelerated atherosclerosis and higher risk of suffering from cardiovascular disease (Yamagishi et al, 2011).

As it has been mentioned before another important molecule with a protective paper against the formation of AGEs, is named PPAR-ү. This is a membrane receptor that acts as a transcription factor regulating the expression of genes. They participate in regulation of vascular tone, inflammation, hypertension, obesity and metabolic syndrome (Oyekan, 2011). PPAR-y reduces blood pressure by reducing the expression of ATII type 1 receptor and inhibiting ATII signaling pathways, what will suppress the renin angiotensin system (RAS). Regulation of hypertension comes from the suppression of RAS and also of the thromboxane $\mathrm{A}_{2}$ system. And by reducing hypertension, renal function is facilitated (Sugawara et al, 2010). PPAR- $\gamma$ agonists could be a powerful tool to improve diabetes complications since its activation also inhibit nitrite, fibronectin and type IV collagen production by mesangial cells and attenuate AGE induced production of IL-8 and ICAM-1 in proximal tubular epithelial cells. PPAR- $\gamma$ agonists also lower TNF- $\alpha$ by suppressing NF$\mathrm{\kappa} \beta$ activation and downregulate RAGEs expression, what in the end leads to an avoidance of the release of ROS, MCP-1 or VCAM-1 (Yamagishi et al, 2011).

AGEs products are really harmful molecules produced in diabetes that can affect to oxidative stress by increasing ROS production and also raise the risk of cardiovascular disease by promoting renal failure and atherosclerosis and the develops of periodontitis.

\section{Advanced glycation end products (AGEs) functions}

- React with macrophages, mesangial cells and produce ROS.

- ROS activate NF- $\kappa \beta$.

- NF- $\kappa \beta$ produce cytokines and growth factors (insulin like growth factor-I, platelet derived growth factor or TGF- $\beta$ ).

- AGEs forms modified collagen that accumulates in blood vessel walls.

- Scavenge LDL and deposit them in blood vessel walls.

- Promotes angiotensin II production.

- Quenching of NO.

Fig. 6. Advanced glycation end products (AGEs) functions 


\section{Conclusion}

So, to summarize, periodontitis not also affects cardiac health in a direct manner by ROS production, but also in an indirect way by promoting the instauration of the metabolic syndrome (obesity, insulin resistance, atherogenic dyslipemia, hypertension, proinflammatory state and prothrombotic state). Even so, the common link between periodontitis and metabolic syndrome or cardiovascular disease is also the action of ROS either acting as a second messenger or directly damaging target molecules as proteins, lipids or DNA. We have seen that periodontitis is a process that generates a great amount of ROS, not only in the site of the infection, the periodontium, but also releases pro-inflammatory cytokines and ROS to the rest of the organism, leading to an oxidative stress situation and a depletion of anti-oxidant molecules over time. This will also contribute to aggravate any other illness that could be already present at the patient or simply help them to arise as any of the metabolic syndrome components. In fact, ROS are seen to participate in lots of organic processes and could be the reason of the instauration of certain afflictions as insulin resistance, hypertension or atheroma plaque development. ROS and oxidative stress are always present at every one of these illnesses that lead to cardiovascular disease, and they even participate directly in cardiovascular damage.

In obesity, hyperglycemia is able to produce ROS by 3 different ways as we saw before: AGEs formation and NF-K $\beta$ activation, conversion of glucose to sorbitol and activation of NADPH oxidase and glucose auto-oxidation. There is also a major metabolic rate in obese people and more NADPH oxidase activity that will produce more ROS than non-obese people. And this higher ROS generation together with the high plasma LDL and free fatty acids levels is able to damage the endothelium by promoting the formation of the atheroma plaque and lipid peroxidation.

In diabetes, AGEs formation produces ROS as in obesity. There is an increased flux in the polyol pathway that produces ROS. Also, diabetic macrophages release more TNF- $\alpha$, IL-6 and IL- $\beta$, what will produce even more ROS. If this is not enough, in diabetes, angiotensin II levels are higher, and we have seen that it is able to produce ROS by stimulation of NADP oxidase.

In hypertension, angiotensin II, endothelin-1 and urotensin II levels are higher, so, they will stimulate NADPH oxidase with its consequents effects over ROS production. These ROS will also uncouple NO synthase and react with NO to form ONOO-. In a hypertensive situation, mitochondria activity is elevated, and therefore, ROS generation will be higher, having higher risk for mitochondrial damage. ROS are also able to produce glomerulopathies and to oxidize LDL thus promoting the formation of atheroma plaque.

Finally, ROS can damage by themselves cardiomyocytes directly, leading to heart malfunction. They also activate matrix metalloproteinases that will remodel coronary blood vessels and arteries, leading to coronary insufficiency. As in hypertension, mitochondrion is also compromised by ROS action, with risk of releasing pro apoptotic molecules that will undergo cardiomyocyte apoptosis. Cardiomyocytes regeneration rate is scarce or even null, so cardiomyocyte loss is a very important event in heart damage and cardiovascular development. 
All these processes implicate ROS production and an oxidative stress situation installed in the organism. We have seen that ROS are involved in lots of damaging mechanisms and they can ease the emergence of some of these afflictions that compromises cardiovascular health. Periodontitis, as a high ROS and pro inflammatory generator process can greatly contribute to aggravate all of these symptoms. It is not involved in cardiovascular failure by itself directly, but in an indirect manner, it can worsen the harmful effects exerted by metabolic syndrome. As we have seen, periodontal treatments decreased ROS generation and the risk of developing some of these illnesses. As these illnesses are risk factors for developing cardiovascular diseases, avoiding periodontitis also lowers the probability of developing these cardiovascular risk factors. This is a good trail that takes us to believe on the relationship between periodontitis and cardiovascular disease.

\section{References}

Ando K, Fujita T. (2009). Metabolic syndrome and oxidative stress. Free Radic Biol Med. 1; 47 (3): 213-8.

Aspriello SD, Zizzi A, Tirabassi G, Buldreghini E, Biscotti T, Faloia E, Stramazzotti D, Boscaro M, Piemontese M. (2011).Diabetes mellitus-associated periodontitis: differences between type 1 and type 2 diabetes mellitus. J Periodontal Res. 46(2): 164-9.

Baelum V, Lopez R. (2004). Periodontal epidemiology: towards social science or molecular biology?. Community Dent Oral Epidemiol. 32 (4): 239-49.

Borges I Jr, Moreira EA, Filho DW, de Oliveira TB, da Silva MB, Fröde TS. (2007). Proinflammatory and Oxidative Stress Markers in Patients with Periodontal Disease. Mediators Inflamm. 2007: 45794.

Borges PK, Gimeno SG, Tomita NE, Ferreira SR (2007). Prevalence and characteristics associated with metabolic syndrome in Japanese- Brazilians with and without periodontal disease. Cad Saude Publica. 23;657-668.

Brecx M, Frolicher I, Gehr P, Lang NP. (1998). Stereological observations on long term experimental gingivitis in man. J ClinPeriodontol 15: (621-627).

Brownlee M. (2001). Biochemistry and molecular cell biology of diabetic complications. Nature. $13 ;$ 414(6865): 813-20.

Bullon P, Morillo JM, Ramirez-Tortosa MC, Quiles JL, Newman HN, Battino M. (2009). Metabolic syndrome and periodontitis: is oxidative stress a common link?. J Dent Res. 88:503-18.

Bullon P, Cordero M, Quiles JL, Morillo JM, Ramirez-Tortosa MC, Battino M. (2011). Mitochondrial dysfunction promoted by Porphyromonas gingivalis lipolysaccharide as a possible link between cardiovascular disease and periodontitis. Free Radical in Medicine \& Biology, in press.

Chang JC, Kou SJ, Lin WT, Liu CS. (2010). Regulatory role of mitochondria in oxidative stress and atherosclerosis. World J Cardiol. 26; 2 (6): 150-9.

D'Aiuto F, Sabbah W, Netuveli G, Donos N, Hingorani AD, Deanfield J, et al. (2008). Association of the metabolic syndrome with severe periodontitis in a large U.S. population-based survey. J Clin Endocrin Metab. 93;3989-3994. 
D'Aiuto F, Nibali L, Parkar M, Patel K, Suvan J, Donos N. (2010). Oxidative Stress, Systemic Inflammation, and Severe Periodontitis. J Dent Res. 89 (11): 1241-6.

Dirkx E, Schwenk RW, Glatz JF, Luiken JJ, van Eys GJ. (2011). High fat diet induced diabetic cardiomyopathy. Prostaglandins Leukot Essent Fatty Acids. May 13. [Epub ahead of print] PMID: 21571515 [PubMed - as supplied by publisher]

Ekuni D, Endo Y, Irie K, Azuma T, Tamaki N, Tomofuji T, Morita M. (2010). Imbalance of oxidative/anti-oxidative status induced by periodontitis is involved in apoptosis of rat submandibular glands. Arch Oral Biol. 55 (2): 170-6.

Fialkow L, Wang Y, Downey GP. (2007). Reactive oxygen and nitrogen species as signaling molecules regulating neutrophil function. Free RadicBiol Med. 15; 42 (2): 153-64.

Fitzgerald DC, Meade KG, McEvoy AN, Lillis L, Murphy EP, MacHugh DE, Baird AW. (2007). Tumour necrosis factor- $\alpha$ (TNF- $\alpha$ ) increases nuclear factor $\kappa B(N F \kappa B)$ activity in and interleukin-8 (IL-8) release from bovine mammary epithelial cells. Vet Immunol Immunopathol. 15; 116 (1-2): 59-68.

Gomes-Filho IS, Freitas Coelho JM, Seixas da Cruz S, Passos JS, Teixeira de Freitas CO, AragãoFarias NS, Amorim da Silva R, Silva Pereira MN, Lima TL, Barreto ML. (2011). Chronic Periodontitis and C-reactive Protein Levels.J Periodontol. 82 (7): 96978.

Houstis N, Rosen ED, Lander ES. (2006). Reactive oxygen species have a causal role in multiple forms of insulin resistance. Nature. 13; 440 (7086): 944-8.

Humphrey LL, Fu R, Buckley DI, Freeman M, Helfand M. (2008). Periodontal disease and coronary heart disease incidence: a systematic review and meta-analysis. J Gen Intern Med. 23 (12): 2079-86.

Karima M, Kantarci A, Ohira T, Hasturk H, Jones VL, Nam BH, Malabanan A, Trackman PC, Badwey JA, Van Dyke TE. (2005). Enhanced superoxide release and elevated protein kinase $\mathrm{C}$ activity in neutrophils from diabetic patients: association with periodontitis. J Leukoc Biol. 78 (4): 862-70.

Kinane DF, Mark Bartold P. (2007). Clinical relevance of the host responses of periodontitis. Periodontol 2000. 43: 278-93.

Kinane DF. (2001). Causation and pathogenesis of periodontal disease. Periodontol 2000. 25: 8-20.

Laky M, Assinger A, Esfandeyari A, Bertl K, Haririan H, Volf I. (2011). Decreased phosphorylation of platelet vasodilator-stimulated phosphoprotein in periodontitis--a role of periodontal pathogens. Thromb Res. 128 (2): 155-60.

Lalla E, Papapanou PN. (2011). Diabetes mellitus and periodontitis: a tale of two common interrelated diseases. Nat Rev Endocrinol. 28. doi: 10.1038/nrendo. 2011. 106.

Liu L, Li Y, Guan C, Li K, Wang C, Feng R, Sun C. (2010). Free fatty acid metabolic profile and biomarkers of isolated post-challenge diabetes and type 2 diabetes mellitus based on GC-MS and multivariate statistical analysis. J Chromatogr B AnalytTechnol Biomed Life Sci. 15; 878 (28): 2817-25.

Marcaccini AM, Meschiari CA, Sorgi CA, Saraiva MC, de Souza AM, Faccioli LH, TanusSantos JE, Novaes AB, Gerlach RF. (2009). Circulating interleukin-6 and high- 
sensitivity C-reactive protein decrease after periodontal therapy in otherwise healthy subjects. J Periodontol. 80 (4): 594-602.

Mealey BL, Oates TW. (2006). American Academy of Periodontology. Diabetes Mellitus and Periodontal Diseases. J Periodontol. 77 (8): 1289-303.

Monteiro AM, Jardini MA, Alves S, Giampaoli V, Aubin EC, FigueiredoNeto AM, Gidlund M. (2009). Cardiovascular disease parameters in periodontitis. J Periodontol. 80 (3): 378-88.

Nassar H, Kantarci A, van Dyke TE. (2007). Diabetic periodontitis: a model for activated innate immunity and impaired resolution of inflammation. Periodontol 2000. 43: 233-44.

Oyekan A. (2011). PPARs and their Effects on the Cardiovascular System. Clin Exp Hypertens. 33(5):287-93

Paquette DW, Brodala N, Nichols TC. (2007). Cardiovascular disease, inflammation, and periodontal infection. Periodontol 2000. 44: 113-26.

Papapanagiotou D, Nicu EA, Bizzarro S, Gerdes VE, Meijers JC, Nieuwland R, van der Velden U, Loos BG. (2009). Periodontitis is associated with platelet activation. Atherosclerosis. 202 (2): 605-11

Paraskevas S, Huizinga JD, Loos BG. (2008). A systematic review and meta-analyses on Creactive protein in relation to periodontitis. J Clin Periodontol. 35 (4): 277-90.

Ramirez-Tortosa MC, Quiles JL, Battino M, Granados S, Morillo JM, Bompadre S, Newman HN, Bullon P. (2010).Periodontitis is associated with altered plasma fatty acids and cardiovascular risk markers. Nutr Metab Cardiovasc Dis. 20 (2): 133-9.

Sugawara A, Uruno A, Kudo M, Matsuda K, Yang CW, Ito S. (2010).Effects of PPARY on hypertension, atherosclerosis, and chronic kidney disease. Endocr J. 57 (10): 847-52.

Sutherland WH, de Jong SA, Walker RJ, et al. (2002). Effect of meals rich in heated olive and safflower oils on oxidation of postprandial serum in healthy men. Atherosclerosis 160(1):195-203.

Tamaki N, Tomofuji T, Ekuni D, Yamanaka R, Morita M. (2010). Periodontal treatment decreases plasma oxidized LDL level and oxidative stress. Clin Oral Investig. 18 Aug 18.

Tonetti MS. (2009). Periodontitis and risk for atherosclerosis: an update on intervention trials. J ClinPeriodontol. 36; 10: 15-9.

Tsai C, Hayes C, Taylor GW. (2002). Glycemic control of type 2 diabetes and severe periodontal disease in the US adult population. Community Dent Oral Epidemiol. 30 (3): 182-92.

Tsutsui H, Kinugawa S, Matsushima S. (2009). Mitochondrial oxidative stress and dysfunction in myocardial remodelling. Cardiovasc Res. 15; 81 (3): 449-56.

Vincent HK, Taylor AG. (2006). Biomarkers and potential mechanisms of obesity-induced oxidant stress in humans. Int J Obes (Lond). 30 (3): 400-18.

Yamagishi SI, Maeda S, Matsui T, Ueda S, Fukami K, Okuda S. (2011). Role of advanced glycation end products (AGEs) and oxidative stress in vascular complications in diabetes. Biochim Biophys Acta. [Epub ahead of print] PMID: 21440603 
Zadik Y, Bechor R, Galor S, Levin L. (2010). Periodontal disease might be associated even with impaired fasting glucose. Br Dent J 208. (10):E20. 


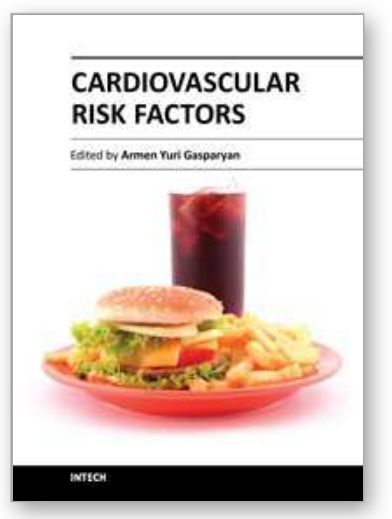

\author{
Cardiovascular Risk Factors \\ Edited by Prof. Armen Gasparyan
}

ISBN 978-953-51-0240-3

Hard cover, 498 pages

Publisher InTech

Published online 14, March, 2012

Published in print edition March, 2012

Cardiovascular risk factors contribute to the development of cardiovascular disease from early life. It is thus crucial to implement preventive strategies addressing the burden of cardiovascular disease as early as possible. A multidisciplinary approach to the risk estimation and prevention of vascular events should be adopted at each level of health care, starting from the setting of perinatology. Recent decades have been marked with major advances in this field, with the emergence of a variety of new inflammatory and immunemediated markers of heightened cardiovascular risk in particular. The current book reflects some of the emerging concepts in cardiovascular pathophysiology and the shifting paradigm of cardiovascular risk estimation. It comprehensively covers primary and secondary preventive measures targeted at different age and gender groups. Attention is paid to inflammatory and metabolic markers of vascular damage and to the assessment of vascular function by noninvasive standardized ultrasound techniques. This is a must-read book for all health professionals and researchers tackling the issue of cardiovascular burden at individual and community level. It can also serve as a didactic source for postgraduate medical students.

\title{
How to reference
}

In order to correctly reference this scholarly work, feel free to copy and paste the following:

Sergio Granados-Principal, Nuri El-Azem, Jose L. Quiles, Patricia Perez-Lopez, Adrian Gonzalez and MCarmen Ramirez-Tortosa (2012). Relationship Between Cardiovascular Risk Factors and Periodontal Disease: Current Knowledge, Cardiovascular Risk Factors, Prof. Armen Gasparyan (Ed.), ISBN: 978-953-510240-3, InTech, Available from: http://www.intechopen.com/books/cardiovascular-risk-factors/relationshipbetween-cardiovascular-risk-factors-and-periodontal-disease-current-knowledge

\section{INTECH}

open science | open minds

\section{InTech Europe}

University Campus STeP Ri

Slavka Krautzeka 83/A

51000 Rijeka, Croatia

Phone: +385 (51) 770447

Fax: +385 (51) 686166

www.intechopen.com

\section{InTech China}

Unit 405, Office Block, Hotel Equatorial Shanghai

No.65, Yan An Road (West), Shanghai, 200040, China

中国上海市延安西路65号上海国际贵都大饭店办公楼405单元

Phone: +86-21-62489820

Fax: +86-21-62489821 
(C) 2012 The Author(s). Licensee IntechOpen. This is an open access article distributed under the terms of the Creative Commons Attribution 3.0 License, which permits unrestricted use, distribution, and reproduction in any medium, provided the original work is properly cited. 